\title{
EVALUACIÓN POR LOGROS
}

\section{Judith de Palacio* \\ Mariela Herrera*}

Un motivo para hacer y presentar este trabajo es que el momento histórico de la humanidad convoca a ser éticos y no lo seriamos si no hacemos explícito nuestro sentir, que sabemos que también es el de muchos maestros, estudiantes, padres, y demás miembros de la comunidad académica, para quienes la evaluación tradicional no da respuesta a sus expectativas como seres humanos. Nuestra experiencia nos ha hecho comprender que el ser humano no quiere ser censurado, calificado y clasificado sino escuchado, tenido en cuenta, como ser humano único.

La idea central de nuestra propuesta es contribuir a evitar que el ser humano se diluya en el afán de lograr objetivos puntuales preestablecidos dejándose de lado: el ser, el sentir, la vida y la existencia. Consideramos que es necesario, que el maestro tenga en cuenta la inclusión de variables o factores que la teoría educativa, construida desde una visión positivista de la educación, no contempla como es el caso del ambiente afectivo de la clase, y la escuela, el saber pedagógico (que no es lo mismo que la acreditación de títulos, aunque no se puede negar que éstos lo influyen) pero que a veces desconocen la experiencia reflexiva del maestro.

\section{ASPECTOS LEGALES SOBRE EVALUACIÓN}

La ley 115 en su Capitulo III, Evaluación, Articulo 80, de conformidad con el articulo 67 de la Constitución Política, plantea que «El MEN con el fin de velar por la «calidad» ${ }^{1}$ y por el cumplimiento de los fines de la educación y por la mejor formación moral, intelectual y física de los educandos establecerá un Sistema Nacional de Evaluación de la Educación (SNEE) que opere en coordinación con el Servicio Nacional de Pruebas del Instituto Colombiano para el Fomento de la Educación Superior, ICFES, y con las entidades territoriales y sea base para el establecimiento de programas de mejoramiento del servicio público educativo.

El sistema diseñará y aplicará criterios y procedimientos para evaluar la calidad de la enseñanza que se imparte, el desempeño profesional del docente y de los docentes directivos, los logros de los alumnos, la eficacia de los métodos pedagógicos, de los textos y materiales empleados, la organización administrativa y física de las instituciones educativas y la eficiencia de la prestación del servicio»

El Decreto 1860 por el cual se reglamenta. parcialmente la Ley 115 en los aspectos pedagógicos y organizativos generales, en su capítulo VI, Evaluación y Promoción, a través de los artículos 47 al 55, establece criterios sobre: evaluación del rendimiento escolar, medios para la evaluación, utilización de los resultados de la evaluación, comisiones de evaluación, registro escolar de valoración, promoción de la educación básica, reprobación, indicadores de logro en la educación básica, indicadores de logro para la educación media.

\footnotetext{
* Profesoras dela Universidad Pedagógica Nacional

${ }^{1}$ Las comillas sonnuestras 
Haciendo una mirada critica a los referentes legales, surgen una serie de interrogantes que inducen a la reflexión sobre la relación entre lo legal y la filosofía de la evaluación por logros. Interrogantes como:

- ¿Qué sentido tiene el diseño de Proyectos Educativos Institucionales bajo una supuesta «autonomía curricular», que pretende responder a necesidades particulares de la comunidad en busca del desarrollo de la misma, si de todas maneras va a existir un SNEE que va a regular y controlar de manera homogénea a todas las instituciones y los seres humanos que las integran?

- ¿Pueden los logros evaluarse bajo indicadores homogéneos, si es que estos se plantean como aspiraciones comunes a nivel nacional, cuando las teorías señalan que las diferencias culturales inciden en las manifestaciones de los logros.

- ¿Cómo hacer concordante los fines de la educación señalados en el Artículo $5^{\circ}$ de la ley 115, basados en principios tales como: autonomía, pluralismo, democracia, convivencia, equidad, respeto a la vida, si el derecho legítimo ala autodeterminación es violentado a través de mecanismos de control que señalan qué lograr y como expresarlo de manera indiscriminada?.

- ¿Cuáles son en realidad los cambios que se pueden dar a través de la aplicación de la Ley 115, y de las disposiciones emanadas de ésta, si parece no existir coherencia entre su filosofía y la propuesta de implementación?

La reflexión sobre los interrogantes anteriores, entre otros, nos lleva a la priorización de la siguiente pregunta:

- ¿Es lo mismo evaluar por logros que evaluar por logros de objetivos?

Intentar dar respuesta a esta pregunta puede ser considerado como el cuerpo fundamental de este trabajo.

\section{ALGO DE HISTORIA}

La evaluación como un acto social universal siempre ha existido, cualquier sujeto la hace en la medida en que no puede dejar de plantearse la cuestión del valor de lo que él mismo u otro está haciendo y su respuesta modifica sus acciones; es decir, está presente implícitamente (Evaluación implícita) y podemos hablar efectivamente de «la omnipresencia de la evaluación en la educación, así como en otros dominios distintos de actividad». (Barbier, 1993, p. 36). Esta forma de evaluación es la base sobre la que se construyen las otras formas de manifestación de la evaluación: la espontánea y la instituida.

La Evaluación espontánea se da cuando en forma natural se manifiesta la propia opinión (juicio de valor) sobre una actividad o sobre una persona. Esta forma de manifestación de la evaluación es extremadamente frecuente, constituye una actividad habitual orientada por criterios que tienen un juicio de "valor" (Barbier, 1993, p.39) que pone en funcionamiento una metodología, unos instrumentos y una tecnología para recoger información y explicitar los datos a fin de ser utilizados. Esta evaluación se considera de carácter científico y su función es clara: "objetivar el proceso de evaluación, realizando la operación con cierta independencia en relación con los actores que la practican” (Barbier, 
1993, p.37). Ejemplos son los exámenes, las calificaciones, los tests, evaluaciones de aptitud, de programas, de métodos, de currículo. Aunque a primera vista se puede decir que la evaluación educativa se ha visto afectada por el desarrollo de las teorías del aprendizaje (conductismo, estructuralismo, constructivismo, ...) y los diferentes enfoques de la educación que éstas provocan, y aunque aparentemente se han dado cambios a nivel de: Quién o qué debe ser evaluado; quién debe evaluar; cómo se deben llevar a cabo las evaluaciones y cómo integrar mejor la evaluación en el proceso educativo, lo que muestra la historia de la evaluación educativa es que desde el momento en que unas prácticas concretas se designan como evaluación, en unas circunstancias y condiciones históricas determinadas, el concepto evaluación se usa para designar prácticas de control denominándose evaluación desde un estado de cuentas hasta el control que un formador (instructor, maestro) ejerce sobre los procesos pedagógicos y a través de éstos al alumno.

Para llevar a cabo el control, se requieren de indicadores en términos de conductas esperadas y previstas dentro del esquema orientación - seguimiento -selección.

Basándonos en el referente conceptual anterior, es posible ubicar la evaluación por logros dentro de una concepcion de evaluación implícita, ya que creemos que como acción inherente a la condición humana, evaluación hacemos todos siempre que reflexionamos sobre nuestra existencia y nuestras acciones lo cual no requiere de ajustarse a las prescripciones de la investigación evaluativa.

La evaluación por objetivos es ubicable dentro del concepto de evaluación instituida que presenta como origen la segunda mitad del siglo XVIII, en donde la presencia de proyectos revolucionarios reclaman por un sistema de enseñanza fundamentado en la apertura a todos para la preparación para funciones especializadas y el reconocimiento de los méritos individuales.

El dispositivo orientación-seguimiento-selección se enfatiza, en el presente siglo, en la década del 30, época caracterizada por la depresión económica y el desempleo, influyendo la educación como mecanismo para discriminar por aptitudes, surgiendo simultáneamente la orientación educativa como forma de seleccionar y eliminar, ya que la orientación no es más que racionalizar la selección para evitar derroches de dinero. El concepto de evaluación como medición, surgida en la década de los años 20, por influencia de la psicometría que pretende perfeccionar la eficacia de los actos de evaluación elaborando instrumentos (los test) para moderar y controlar los juicios de valor, conducen a una estandarización de todos los componentes de la educación. Bajo este paradigma, las variables para las que no se poseen instrumentos de evaluación y escalas de medición, corno los afectos y valores, quedan por fuera de la evaluación ya que el dato, recogido a través de tests, se puede procesar y analizar estadísticamente para tomar decisiones y establecer normas considerándolos por esto útil. Se asume entonces la curva normal como expresión matemática del orden natural.

Posteriormente, década del 40 , la evaluación se concibe como la comprobación de la congruencia entre resultados y objetivos. Dichos objetivos están referidos a cambios predeterminados como deseables en la conducta del individuo. La filosofía y psicología a través de la cual se implementó este concepto fue la conductista, razón por la cual los objetivos se expresaron en términos de conductas observables, tomándose éstas como criterio único, pasando a ser la evaluación una técnica terminal de comprobación de productos dejando de lado las interacciones sociales y culturales, ocurridas dentro y fuera del aula, cuyos resultados también pueden ser evaluados así no se hayan incluido expresamente en los planes de estudio y programas. 
Durante la década del 50 , en el marco de la industria, se plantean por primera vez los problemas de evaluación de acciones (para nosotras evaluación de actuaciones) ya no solo de conductas, y se privilegia la evaluación como elemento importante para establecer relaciones cuando existe un vinculo contractual. Este modelo evaluativo es trasladado al campo de la educación lo cual es obvio si se tiene en cuenta el encargo social que a la educación se hace, dc mantener el vinculo entre el modelo de desarrollo socio-económico, que esté vigente, y los procesos de formación.

En las décadas del 60 y 70 toma auge la evaluación en términos cibernéticos, basada en la noción de sistema y la noción de información. A esto tampoco escapa el sistema educativo, en donde la evaluación es entendida como el proceso de recoger información y utilizarla en las tomas de decisión. Ejemplo de esto son los modelos IPP (Insumo, Proceso, Producto) y CIPP (Contexto, Insumo, Proceso, Producto).

Actualmente se observa cambio en la evaluación, pues ya no solo se evalúa el estado final del perfil de capacidades, presentado por un individuo, al termino de un proceso de formación (evaluación sumativa) sino que se evalúan los estados sucesivos de ese perfil a lo largo del proceso (evaluación formativa) de tal forma que el maestro puede intervenir en la trayectoria de los sujetos en formación y ejercer un control continuo, lo que se asume como la función educativa de la evaluación. Otro cambio que se observa es la apertura de un espacio de autonomía o de libertad funcional para los individuos implicados en la evaluación (Barbie, 1993, p. 57) quienes pasan, previa asimilación de los roles que se espera de ellos, a ser evaluadores de si mismos, es decir, entran a «jugar el juego de la participación» sustituyendo los controles externos por los «autocontroles». Dentro de estos cambios de la evaluación, parece ser que se encuentran la propuesta de evaluación por logros y los diseños de PEI, pero que en última instancia obedecen a la presión ejercida por actores externos a la acción evaluada y que regularmente administran el gasto, determinan las políticas educativas y orientan a través de lineamentos generales la planificación de la enseñanza, ejemplo: el plan decenal y los lineamientos generales del currículo.

Desde esta mirada sucinta a la historia de la evaluación instituida, creemos haber caracterizado la evaluación por objetivos. Convencidas de que evaluar por objetivos no es lo mismo que evaluar por logros, intentaremos caracterizar esta última.

Como primera afirmación planteamos que objetivos y logros no son lo mismo, por lo tanto las formas de evaluación correspondientes a cada caso no pueden ser iguales, de no ser que por evaluación por logros se esté entendiendo evaluación por logros de objetivos.

Quien evalúa por objetivos determina de antemano lo que el alumno debe hacer, mostrar, evidenciar, y junto a ello debe plantear las condiciones de ejecución. Quien evalúa por logros, ha de ser consciente de que no es posible predeterminar, ni prever, las manifestaciones de los logros, pero que debe conocer los procesos que se generan en la consecución de un logro para identificar sus manifestaciones como expresión de que dicho proceso se ha dado, es decir, las manifestaciones de los logros no se reducen a la evidencia de transformaciones externas de conducta, como las planteadas por los objetivos. Las manifestaciones de los logros son sus indicadores, que pueden ser dados en diferentes niveles en un grupo escolar, pues es necesario reconocer que un estudiante siempre logra los propósitos del programa pero lo que varia es el nivel de logro. 
Tanto en la evaluación por objetivos como en la evaluación por logros se reconoce que se dan cambios de conducta y comportamientos, la diferencia está en los caminos que se recorren para alcanzarlos. En el caso de los objetivos, los cambios se esperan como finalidad del aprendizaje. En el caso de los logros, los cambios se dan como consecuencia de procesos.

Lograr algo significa sentir que se han hecho progresos con respecto a algo que se intenta y se desea, los logros son de carácter personal, son siempre percibidos por el actor y dependiendo de su nivel son susceptibles de ser percibidos por los demás. Por lo tanto, mientras que la evaluación por objetivos se basa en la aplicación de pruebas, la evaluación por logros se basa en la observación y el diálogo entre maestros-estudiante, desbordando el concepto de diálogo verbal, trascendiéndolo a diálogos observacionales, a diálogos introspectivos.

La evaluación por logros devuelve al sujeto evaluado el rescate de su potencialidad de decidirse y pensarse (base de la autonomía) mientras que la evaluación por objetivos le restringe esta facultad, pues siempre esta siendo decidido y pensado por otros.

El atreverse a asumir cada quien su autonomía, su autodeterminación, va a originar cambios actitudinales que quienes ostentan el poder no pueden predeterminar acostumbrados y confiados como están a la manipulación y abuso que en aras de la ley, la ciencia, la tecnología y Dios han tenido.

La evaluación por objetivos es fácilmente controlable pareciendo ser que su alcance depende fundamentalmente del individuo, aislado de sus contextos particulares. La evaluación por logros toma en consideración la historia de vida de cada individuo y los contextos en que este se desempeña y vive, asumiendo como retos la superación de obstáculos que estos contextos puedan presentar para los logros deseados.

La evaluación por logros no puede verse obstaculizada por eventos tales corno falta de recursos, de capacitación, sin que esto signifique exonerar al Estado de sus obligaciones. La transformación de la evaluación depende mas de cambios de actitud del maestro y de todos los actores de la educación, en sus formas de pensar el mundo, al ser humano y la vida. Dichos cambios se traducen en tomas de decisiones que son arriesgadas y transgresoras del statu quo. Arriesgadas en el sentido de cambios radicales como por ejemplo en la forma de organización de horarios y contenidos, pasando de un modelo en el que se secciona tanto el tiempo corno los saberes a un modelo integrador. Cuando una comunidad educativa se propone unos logros y adquiere compromiso frente a ellos, el trabajo es generar las condiciones propicias o adecuadas, es hacer posible lo imposible aún dentro del modelo capitalista vigente.

\section{A TÍTULO DE SUGERENCIAS}

Pasar de un sistema de evaluación por objetivos a un sistema de evaluación por logros no es fácil.

Todo proyecto educativo involucra una evaluación. Pero subyacente a cada forma de evaluación existe una concepción de: educación, proyecto de desarrollo humano, modelo pedagógico, los cuales a su vez implican conceptualizaciones de: hombre, cultura, sociedad, ser, tener, sentido de la vida, entre otros. 
Pasar a un modelo evaluativo por logros exige una mirada crítica a los conceptos vigentes anteriormente mencionados, así por ejemplo, dentro del sistema actual de educación, son consideradas como académicamente importantes las asignaturas que buscan la articulación de la educación con la economía. Las demás, son consideradas y de nominadas dentro de la cultura académica tradicional como vocacionales, costuras, coprogramáticas, extraeurriculares e incluso en una época como medias materias y no causal de pérdida del año escolar. Estas materias han sido las correspondientes al área de la educación estética (danza, música, dibujo, cerámica, teatro, ...), manualidades (trabajo con papel y cartón, costura, telares y tejidos, ...), mecanografía, culinaria y por supuesto Educación Física. Como si sus esencias y sentidos no fueran parte de la constitución del ser humano, sino agregados, olvidando que fueron justamente éstas quienes permitieron la evolución del hombre, lo llevaron a la cumbre de la razón y que su descuido y comercialización lo han llevado al estado lamentable de actual deterioro, porque con estas experiencias también se le ha quitado la posibilidad de ser, es decir de autoconstituirse y de interconstruirse.

Dentro de un concepto de evaluación por logros, la imposición y predeterminación de indicadores no tienen sentido, y esto no quiere decir que rechacemos la idea de tener horizontes, propósitos, sino que al maestro lo vemos como propiciador, creador de ambientes, colaborador, cooperador, demócrata, y al alumno como autogestor y actor de su propio aprendizaje, pero como libretista y director y no solo como actor ingenuo que memoriza el libreto. El acto de enseñanza-aprendizaje se vuelve así creación colectiva y por lo tanto los logros pueden ser propuestos por los estudiantes, por los maestros, concertados entre estos dos grupos y entre estos y el establecimiento.

Es necesario que el maestro tome conciencia de que:

- Permitir que se nos decidan y homogenicen los indicadores de logro es aceptar que no es capaz de asumir compromiso ético-político y decidir el tipo de colombiano que el país necesita y trabajar por un proyecto de desarrollo humano acorde con la exigencias sociales incluidas en éstas las constitucionales.

- Pretender evaluar por logros en un sistema cuyos ejes son la eficiencia, la eficacia y la competencia, reguladas por la lógica del mercado, es una contradicción que solo puede ser superada, en la medida en que tengamos claridad conceptual sustentada en referentes históricos y epistemológicos que permitan establecer argumentos y precisiones en el proceso de tomar decisiones; así por ejemplo debemos estar alerta para comprender que al hablarnos de indicadores de logros de objetivos nos pueden confundir: logro es deseo, pero este concepto se quiere cambiar por el de logro es conducta observable, así mismo, la Ley 115 nos habla de tolerancia y de democracia, ¿pero cómo va a existir tolerancia con la competencia y democracia con el individualismo que fundamentan el modelo de desarrollo social vigente?.

Los indicadores de logro, como manifestaciones, evidencian señales de procesos que no deben ser reducidos al estrecho marco de una disciplina, ya que un indicador de logro es producto de la integración de saberes académicos, sociales y culturales. Los indicadores de logro no excluyen la presencia de eventos que pongan en evidencia el trabajo realizado tales como: informes, videos, narraciones, conferencias, dramatizaciones, diferentes expresiones artísticas que ayudan a demostrar que si es posible transformar la realidad modificando la cultura y la sociedad. 
La evaluación ha de ser pensada como un hecho importante no solo dentro del contexto de la institución escolar, sino a lo largo de la vida. El ser humano siempre tiene aspiraciones, deseos, metas, frente a esto la evaluación por logros podrá ser más elemental pero es mas humana. La evaluación por logros es implícita ala condición de ser humano.

A partir de las reflexiones presentadas hasta el momento, hemos construido un paralelo entre las características de la evaluación por objetivos y de la evaluación por logros.

\begin{tabular}{|l|}
\hline Evaluación por objetivos \\
\hline $\begin{array}{l}\text { Los objetivos son adquisiciones y deseos } \\
\text { vendibles }\end{array}$ \\
\hline
\end{tabular}

Hay un punto mínimo cuantitativo para ser promocionado. Hay linealidad en el ascenso formal lo que implica progreso en una sola dirección, un hombre parcial. Hay prerrequisitos que bloquean y condicionan la participación.

El modelo de desarrollo actual se basa en la eficiencia, la eficacia y la competencia e implica la inmersión en el modelo educativo tecnológico que busca la formación de un hombre reactivo y productivo en algunas de las fases del proceso industrial. No es gratuito que la educación utilice vocabulario de fábrica (área, sector, producto, criterios de calidad).

Existen diseños de programas secuenciales inter e intra asignaturas que obedecen a la estructura disciplinar y a los intereses del mercado. Bajo este paradigma la conducta es consecuencia del objetivo formulado

El profesor y el estudiante son decididos por fuerzas externas desconocidas para ellos que operan como leyes naturales.

Requiere de formular políticas y principios filosóficos frente a las órdenes del Estado. Para ello hay una búsqueda de desarrollo de un proyecto de modelo político y social que no necesariamente implica la consideración de un proyecto de desarrollo humano distinto de aquel que satisface sus intereses.

El modelo debe contemplar los mecanismos de control para avalar los logros sociales educacionales, de promoción y titulacíon.

\section{Evaluación por logros}

Los logros son consecuencias de vivencias y deseos reales

Hay avance, no hay punto minimo cuantitativo para ser promocionado lo que implica modificar las condiciones administrativo académicas de la institución escolar. No hay prerrequisitos, hay integración, se participa, se es, se construye.

Busca el desarrollo de mi potencialidad.

Se parte de unos propósitos y los programas se diseñan conjuntamente por maestros y estudiantes según las necesidades y deseos, lo cual no implica caos, es decir que los logros son consecuencia de los propósitos.

El maestro y el estudiante toman decisiones y procuran develar las fuerzas externas que inciden las mismas. Ejemplo, diseñar canchas sin líneas demarcatorias.

Requiere de señalar políticas y principios filosóficos frente al ser humano. Evaluar por logros surge de una concepción de desarrollo humano no necesariamente colgada del modelo de desarrollo social impuesto por el Estado.

Aunque el modelo se centra en los logros individuales, y no enfatiza en los mecanismos de control externo, debe presentar ideas para determinar estrategias de negociación con os logros sociales ya que los dos tipos de logros son importantes. 
Las responsabilidades frente a los logros de Las responsabilidades frente a los logros objetivos son asignadas y distribuidas así: son compartidas y se dan por compromiso.

unas corresponden a los maestros, otras a los alumnos, otras a la administración y otras al Estado.

\begin{tabular}{|l|lr|}
\hline Hay resultados predecibles. Evalúa & Hay resultados esperados, pero también \\
conductas o comportamientos externos \\
predeterminados por el maestro, los cuales \\
hay otros inesperados, hay magia. \\
intenta homogeneizar y masificar
\end{tabular}

El alumno es medido con base en El estudiante da cuenta de si a medida que estereotipos o modelos y los resultados se expresa descriptivamente los logros expresan numéricamente. Se basa en la alcanzados. Funciona como un proceso en utilización de técnicas de recolección de in el cual hay un caer en cuenta, un reconocer, formación para emitir juicios de valor acerca hasta donde he desarrollado mi potencial de los resultados obtenidos por el sujeto que humano. Asume que una fuente importante es sometido a la evaluación para ser de información es el sujeto mismo calificado y clasificado. Enfrenta todos los quien es ayudado, orientado, pero que problemas inherentes a los problemas de siempre es autónomo y libre de aceptar las validez y confiabilidad de los datos de sugerencias. Los problemas de validez y información, así como a su procesamiento y confiabilidad de los datos de información, análisis.

así como a su procesamiento y análisis, quedan sin sentido

La relación profesor-alumno es vertical y La relación maestro - estudiante es normativa, en la cual uno y otros tienen que horizontal - circular enfocados todos hacia el esforzarse por lograr objetivos ajenos a su logro de motivos comunes y/o personales, sentir, de los cuales muchas veces no se escogidos por ellos y según reglas comprende su utilidad ni su sentido acordadas entre todos.

El trabajo académico se puede quedar dentro Normalmente las condiciones del trabajo de los limites topográficos del lugar de la provocan una integración con la comunidad, escuela. Acorde con ello, la evaluación tiene ampliándose así el espacio escolar. Acorde lugar y tiempo determinado al interior del con ello, la evaluación es permanente y espacio escolar.

Los proyectos son predeterminados desde afuera

continua y no tiene lugar ni tiempo determinado, se da en el espacio de la vida

Focaliza la observación en aspectos Observa al hombre total, no puede darse de particulares de las dimensiones del ser, así otra forma. Logros internos y externos son evalúa por separado lo psicomotor, lo importantes, lo malo es trabajar polarizado afectivo, lo cognoscitivo

sin un énfasis en los procesos.

Plantea en la descripción del objetivo la Plantea propósitos y metas alcanzables en conducta esperada y las condiciones de ej corto piazo pero deja abierto el espacio ejecución de esa conducta, así corno el para la diferencia de caminos y formas de criterio de aceptabilidad.

lograrlos, luego hay creatividad y sorpresa y los actores son capaces de identificar sus logros y de dar cuenta de ello.

La evaluación por objetivos exige del La evaluación por logros exige del maestro maestro conocimiento de las formas externas conocimiento de procesos de desarrollo y de la conducta, por lo cual a éste le hasta un bagaje cultural lo suficientemente rico con conocer los cornos operativos para que le permita interpretar y asistir al establecer indicadores y no tiene que debatir estudiante.

su saber pedagógico. 


\section{BIBLIOGRAFÍA}

BARBIER, Jean - Marie. La evaluación en los procesos de formación. Barcelona, Paidós, 1993.

FECODE. Educación y Cultura. (Revista numero 39). Santafé de Bogotá, marzo de 1996

FROMM. Erich. ¿Tener o ser?. Colombia, Fondo de Cultura Económica 1994.

LAFOURCADE, Pedro D. La evaluación en organizaciones educativas centradas en logros. Méjico, Trillas, 1982.

NIÑO, Libia Stella, et al. Evaluación, proyecto educativo y descentralización en la educación. Santafé de Bogotá, UPN, 1995.

PAREDES, Ligia. Seminario Taller sobre evaluación educativa. Bogotá, UNISUR, 1988.

REPUBLICA DE COLOMBIA. Ley General de Educación. Santafé de Bogotá, EI Pensador, 1995.

STUFFLEBEAM, Daniel, et al. La evaluación educativa. Bogotá, Pontificia Universidad Javeriana, 1982

UNIVERSIDAD PEDAGÓGICA NACIONAL. La problemática de la evaluación escolar en Colombia. Bogotá, UPN, 1987. Santa Marta, Junio 27 - Julio 1 de 1996 